\title{
THE BOUNDARY INTEGRAL EQUATION METHOD WITH APPLICATION TO CERTAIN STRESS CONCENTRATION PROBLEMS IN ELASTICITY
}

\author{
F. J. RIZZO and D. J. SHIPPY
}

(Received 7 October 1980)

\begin{abstract}
The essential aspects of the Boundary Integral Equation Method for the numerical solution of elliptic type boundary value problems are presented. A numerical example for a stress concentration problem in classical elasticity in three dimensions is given along with several examples for a class of scalar problems in elastic torsion of non-cylindrical bars. Some discussion and criticism of the method itself and in comparison with more widely used field methods is also presented.
\end{abstract}

\section{Introduction}

This paper is an account of what are, in the authors' view, the essential aspects of the currently popular Boundary Integral Equation (BIE) method for the numerical solution of a variety of problems in applied mathematics and engineering. Emphasis here is on problems governed by systems of linear elliptic partial differential equations. The BIE method has been used successfully for parabolic and hyperbolic problems, and, to some extent, for nonlinear problems in an incremental fashion (see for example [20], [1]). However, many, if not most, of the current applications of the BIE method are for nontransient field problems involving potential distributions or elastic stress. Such problems are elliptic and the easiest for which to describe the essential BIE machinery. Results from several illustrative numerical experiments are included.

Specifically, Sections 2 and 3 contain concise but reasonably self-contained descriptions of a BIE formulation and a modern numerical solution process.

OCopyright Australian Mathematical Society 1981 
Section 4 contains results from recently completed research on a BIE solution for the elastic stress concentration in a perforated plate in bending. This problem, in three spatial dimensions, requires, as is inherent in the method, only surface discretization, and is one of a type for which a BIE treatment is perhaps used to best advantage. Section 5 contains results for several stress-concentration problems in the theory of torsion of axially symmetric elastic shafts. This class of problems, although governed by a single scalar equation, rather than a vector set as for the problem of Section 4, involves a linear operator with non-constant coefficients. The fundamental solution, only recently obtained, required for the BIE formulation is a comparatively complicated complete elliptic integral.

Finally, Section 6 contains some discussion and criticism of the BIE method together with certain comparisons with field numerical methods.

\section{BIE formulation}

Consider the partial differential equation

$$
L(w)=0 \text { in } B,
$$

where $B$ is a region of two $\left(E^{2}\right)$ - or three $\left(E^{3}\right)$-dimensional space with surface $S$, and $L$ is a linear elliptic differential operator of order $m$ with analytic coefficients operating on a sufficiently smooth scalar function $w$ of variables $x \equiv$ $\left(x_{1}, \ldots, x_{n}\right), n=2$ or 3 . Green's reciprocal identity for the operator $L$ may be written

$$
\int_{B}[u L(v)-v \bar{L}(u)] d B=\int_{S} M_{v}(u, v) d S,
$$

in which $u$ and $v$ are arbitrary functions, $\vec{L}$ is the operator adjoint to $L$, and $M_{v}$ is a bilinear differential operator of order $m-1$, obtainable from $L$, and which depends on the outward normal $\nu$ to $S$ (see, for example, [9]). All geometry is assumed sufficiently regular (in the sense of Kellogg [10]) to permit application of the divergence theorem. Now if $v$ is chosen to be the fundamental solution $K(x, y)([9$, Chapter 3]) of (2.1) such that

$$
L[K(x, y)]=\delta(x-y),
$$

where $x \in B, y \in B$ or $S$ and $\delta$ is the Dirac delta function, then (2.2) gives rise to

$$
u(x)=\int_{S} M_{\nu}[u(y), K(x, y)] d S(y)+\int_{B} K(x, y) \vec{L}[u(y)] d B(y),
$$

where we have used the sifting property (see Stakgold [21]) of the delta function. 
Next, suppose $L$ is self adjoint and $u$ is a regular solution of (2.1) so that the $B$ integral in (2.4) vanishes. The remaining integrand may be expanded and an appropriate limit process (Muskhelishvili [14]) performed to permit interpretation of the $S$ integral when $x$ as well as $y$ is on $S$. Under these conditions, (2.4) leads to

$$
c(x) u(x)=\int_{S}\left\{u(y) B_{v}[K(x, y)]-K(x, y) B_{p}[u(y)]\right\} d S(y)
$$

in which the operator $B_{v}$, obtainable from $M_{v}$ and of the same order, may be regarded as a generalized normal derivative. Further, $c(x)$ is a coefficient with value unity for $x \in B$, zero for $x$ in the region exterior to $B$, and one-half for $x \in S$, provided $S$ has a unique tangent plane at $x$. Otherwise, $c(x), x \in S$, is given by (2.5) using $u=$ a known elementary solution of (2.1).

Equation (2.5) is the formula upon which a BIE solution for boundary value problems governed by (2.1) may be based (see [8] and [16] for early examples for classical $L$ 's). Note first that a well posed problem governed by (2.1) requires that $u$, or $B_{v}(u)$, or a suitable linear combination of each function be prescribed over $S$. Different prescriptions of such data may be made over parts of $S$ to describe so-called mixed problems. However, regardless of the type of problem, $u$ and $B_{y}(u)$ are never simultaneously known in advance at arbitrary $y$ on $S$. Thus, either the first or second integral in (2.5) remains unknown over $S$ (or parts of $S$ for mixed problems) following prescription of boundary data.

The solution strategy then, for $u(x), x \in B$, via (2.5) is as follows. Choose $x$ and $y$ on $S$ in (2.5) and regard that equation as a constraint between the pair $\left\{u, B_{v}(u)\right\}$, defined on $S$, pertaining to one and the same function $u$ defined in $B$ satisfying (2.1). Moreover, regard (2.5) as a BIE to be solved for that part of the pair $\left\{u, B_{v}(u)\right\}$ not known from the prescription of boundary data. Once this integral equation is solved such that both parts of the pair $\left\{u, B_{v}(u)\right\}$ are known everywhere on $S$, choose $x \in B$ in (2.5) and generate $u$ at any desired $x \in B$ to complete the solution process.

If desired, gradients of $u$ at $x$ may be obtained by differentiation of the integrand in (2.5), where we note that only the fundamental operator-dependent functions $K(x, y)$ and $B_{y}[K(x, y)]$ are affected since they alone are functions of $x$ in (2.5).

Some remarks concerning the above approach and similarities with and departures from classical integral approaches, with which the reader is undoubtedly familiar, will be made in the closing section. Clearly, however, a strictly analytical solution to a BIE arising in any practical problem is entirely out of the question. Some systematic numerical solution procedure of the type outlined in the next section is in order. 


\section{A numerical procedure}

To numerically implement the BIE solution strategy, imagine that $S$ of $B$, $B \in E^{3}$ is discretized into a number $M$ of curvilinear surface elements of either quadrilateral or triangular shape, and locate eight or six nodes, respectively, on each element. Half of the nodes on each element are at the vertices and the other half are on the curvilinear edges, usually midway between the vertex nodes. The coordinates $y_{i}^{\alpha}$ of each node are specified, and the coordinates of a non-nodal point of an element are assumed to be given by ${ }^{\dagger}$

$$
y_{i}(\xi)=M^{\alpha}(\xi) y_{i}^{\alpha}, \quad \text { for } \alpha=1,2, \ldots, 6 \text { or } 8 \text {, and } i=1,2,3 \text {, }
$$

in which $M^{\alpha}(\xi)$ are second-order shape functions of intrinsic coordinates $(\xi) \equiv$ $\left(\xi_{1}, \xi_{2}\right)$ of the type in familiar use in finite element analyses (see for example Zienkiewicz [27]). The geometry of $S$ is thus approximated by a system of piecewise quadratic curvilinear elements coincident at the nodes. Next, assume that any of the functions of $y$ alone in (2.5) varies over the elements according to

$$
\phi(\xi)=M^{\alpha}(\xi) \phi^{\alpha},
$$

where $\phi^{\alpha}$ is the nodal value of the particular variable at issue. Thus, the functions $u(y)$ and $B_{\nu}[u(y)]$ are approximated by piecewise quadratic polynomials over the elements.

Under these basic discretizing assumptions, (2.5) for $x \in S$ becomes

$$
\begin{aligned}
c\left(x_{\eta}\right) u\left(x_{\eta}\right)= & u^{\alpha \sigma} \int_{\gamma} M^{\alpha}(\xi) K^{\prime}\left[x_{\eta}, y(\xi)\right] J^{\sigma}(\xi) d \xi \\
& -u^{\alpha \sigma} \int_{\gamma} M^{\alpha}(\xi) K\left[x_{\eta}, y(\xi)\right] J^{\sigma}(\xi) d \xi,
\end{aligned}
$$

in which the notation $w^{\prime} \equiv B_{\nu}(w)$ is introduced for brevity, $\sigma=1,2, \ldots, M$, and $\eta=1,2, \ldots, N$, where $N$ is the total number of nodes on $S$. The quantities $\phi^{\alpha \sigma}$ mean "the value of $\phi$ at local node $\alpha$ on surface element $S_{\sigma}$ ", and $J^{\sigma}(\xi)$ is simply the ratio $d S_{\sigma}(y) / d \xi$ for a particular element. Note too that (3.1) is the function which maps $S_{\sigma}$ to the standard shape $\gamma$ which is either a square or equilateral triangle in the space $\xi_{1} \xi_{2}$.

The integrals in (3.3) involve only known integrands now, and although certain of them are improper whenever $x_{\eta} \in S_{o}$, and indeed sometimes have meaning only in the sense of the Cauchy Principal Value, all are integrable either analytically or by Gaussian quadrature following removal of the singularity via transformation (see for example [11] or [18] for details). In any case, adopting a global designation for $\phi^{\alpha \sigma}$, and letting $x_{\eta}$ occupy all of the nodes,

\footnotetext{
$\uparrow$ Summation is implied on repeated indices $\alpha$ and $\sigma$ only.
} 
(3.3) is a square $N \times N$ system of linear algebraic equations of the form

$$
\mathbf{A u}=\mathbf{B} \mathbf{u}^{\prime},
$$

in which the square matrices $\mathbf{A}$ and $\mathbf{B}$ contain the known integrals (and the $c\left(x_{\eta}\right)$ term) in (3.3), and the columns $\mathbf{u}$ and $\mathbf{u}^{\prime}$ contain the globally numbered nodal values of the original function pair $\left\{u, B_{v}(u)\right\}$.

The continuous BIE (2.5) for $x \in S$ is thus approximated by (3.4). A numerical solution to a given boundary value problem then requires an appropriate combination of the pair $\left\{\mathbf{u}, \mathbf{u}^{\prime}\right\}$ to be specified, and (3.4) is solved for the nonspecified part of the pair $\left\{\mathbf{u}, \mathbf{u}^{\prime}\right\}$. Once this is done, an "interior version" of (3.3) is used to generate $u(x)$ pointwise at chosen $x \in B$ to complete the solution. Note that for $x \in B$ the integrals in (3.3) are all proper.

Often, however, only the boundary solution is of interest, such that nonspecified $\mathbf{u}^{\prime}$ or $d \mathbf{u} / d S$ on $S$ are all that are required as is the case with the examples to follow.

For $B \in E^{2}, S$ is a curve or union of several curves and the preceding remarks apply essentially intact with an appropriate reduction in dimension. Note that $S_{o}$ is now an element of boundary curve, with nodes at each end and one (usually midway) between. Now there are three shape functions $M^{\alpha}(\xi)$, $\alpha=1,2,3, \xi$ is a single scalar variable, and $\gamma$ is simply the interval -1 to 1 (see $[5])$.

\section{An elasticity problem}

The ideas in the preceding sections may be generalized so that $L$ is a vector operator, $u$ and $B_{\nu}(u)$ are vector variables, $K(x, y)$ and $c(x)$ are multi-dimensional vectors (or tensors) as required, and the terms in (2.5) involve the appropriate inner product of functions to yield a vector function on both sides of the equation.

Specifically, a vector equation of considerable practical interest is the Navier equation (Love [12]) governing problems in classical elastostatics, that is

$$
L(u) \equiv(\lambda+u) \operatorname{grad} \operatorname{div} u+\mu \operatorname{div} \operatorname{grad} u=0,
$$

in which $u$ is the elastic vector displacement field and $\lambda$ and $\mu$ are constants. Following the ideas in Section 2, using $U(x, y)$, the fundamental Kelvin solution of (4.1) (see Appendix), the counterpart of (2.5) has the form

$$
c(x) u(x)=\int_{S}[t(y) U(x, y)-u(y) T(x, y)] d S(y),
$$


in which $t$ and $T$ are, respectively, $B_{\nu}(u)$ and $B_{\nu}(U)$ where

$$
B_{\nu}(u) \equiv \lambda(\operatorname{div} u) \nu+\mu\left[\operatorname{grad} u+(\operatorname{grad} u)^{T}\right] \nu .
$$

Physically, $t$ is the surface traction vector applied to $B$ across $S$ to induce the stress field of interest.

Consider the specific problem of an elastic plate with dimensions $10 \times 16 \times 4$ perforated with a hole of unit radius. Fig. 1 shows (to scale) one quarter of this piate together with the surface eiement discretization pattern used to obtain a numerical solution via the process of Section 3. The plate is loaded in lateral bending by applying a linearly distributed bending traction (component of $\mathbf{u}^{\prime}$ ), the tensile part of which is as shown in Fig. 2. The output for this problem, the normal stress distribution (part of nonprescribed $\mathbf{u}^{\prime}$ ) along the midsurface of the hole is shown (tensile part only) in the same figure.

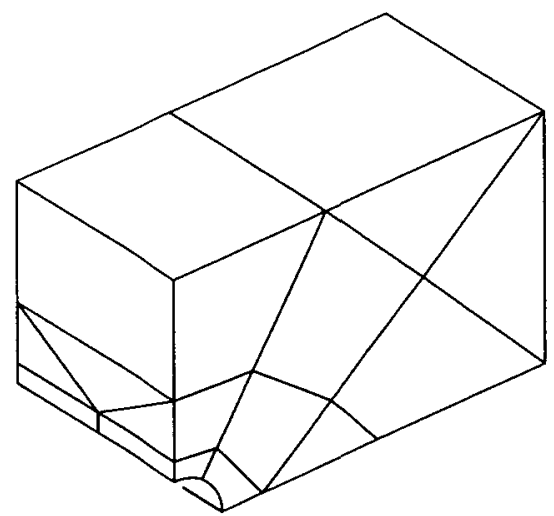

Fig. 1. Discretization of perforated plate.

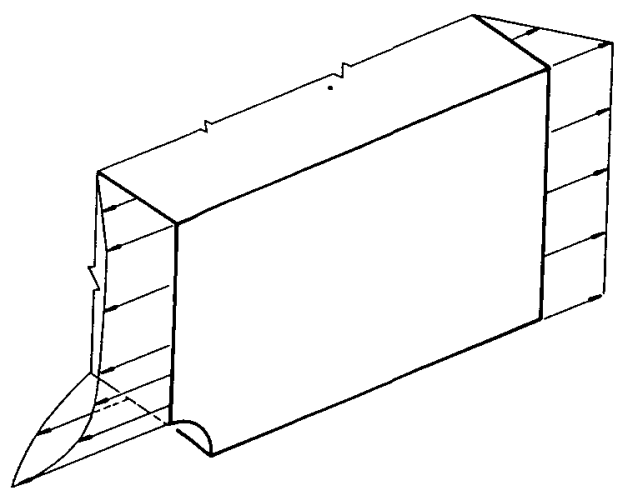

Fig. 2. Stress distribution for perforated plate. 
Using $M=33$ elements with $N=100$ nodes the stress concentration, defined as the ratio of the peak stress at the hole to the nominal bending stress, was obtained from our solution to be 2.49 . This compares favorably with an expected value from the literature $([15$, p. 231]) of 2.62 . The computations involved $3 \times N=300$ simultaneous equations and were made on an IBM $370 / 165$ computer in $193 \mathrm{sec}$ CPU time.

\section{An axisymmetric torsion problem}

Consider the class of problems governed by the scalar equation

$$
L(v) \equiv r \Delta v+\frac{\partial v}{\partial r}-\frac{v}{r}=0
$$

with

$$
\Delta \equiv \frac{\partial^{2}}{\partial r^{2}}+\frac{\partial^{2}}{\partial z^{2}}
$$

where $v=v(r, z)$ is the transverse component of displacement of a point in an elastic bar of circular cross-section, but variable diameter, subjected to torsion; $r$ and $z$ are radial and axial coordinates, respectively. The maximum shear stress $\tau$ at the surface of such a bar is usually of interest and is given by

$$
\tau=\mu r d \psi / d S,
$$

in which $\psi \equiv v / r$ and $\mu$ is one of the elastic constants (shear modulus) referred to earlier. Again, following the ideas in Section 2, the appropriate BIE for this class of problems may be written (see [17], compare with [3])

$$
c(x) r(x) \psi(x)=\int_{S}\left[\frac{\partial \psi}{\partial \nu}(y) K_{1}(x, y)-\psi(y) \hat{K}_{1}(x, y)\right] r(y) d S(y),
$$

in which $K_{1}(x, y) / r(y)$ is the appropriate fundamental solution of (5.1) with property (2.3). (Explicit forms of $K_{1}$ and $\hat{K}_{1}$ are given in the Appendix.)

Consider the problem involving a grooved shaft as depicted in Fig. 3. We seek the stress concentration $k$ at the surface of the groove, defined to be the ratio of the maximum surface shear stress to the nominal surface shear stress on a uniform shaft of the smaller diameter $d$. For $D / d=1.05$ and four values of the groove radius, $a$, as depicted, the computed values $k_{\text {BIE }}$ are compared with accepted values $k_{T}$ in Fig. 3. The domain of definition of the function $v$ is the axial cross section depicted in the figure, the boundary of which was discretized using 25 nodes for each problem. A more stringent test is provided by considering a more deeply grooved shaft (not shown) with dimensions $D=10, d=8$, and $a=0.1$. Here, a total of 61 nodes was used, 13 of which were on the semicircular groove, and the $k_{\mathrm{BIE}}=3.11$ so obtained compares favorably with the $k_{T}=3.10$ value given by Rushton [19]. 


\section{Grooved Shaft}

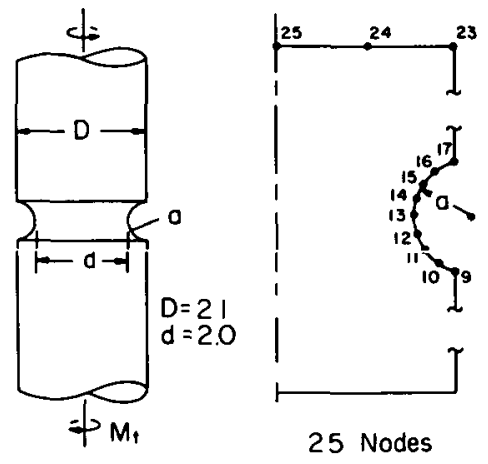

\begin{tabular}{|l|l|l|}
\hline 0 & $k_{\text {BIE }}$ & $k_{T}$ \\
\hline .4 & 1218 & 123 \\
.2 & 1337 & 135 \\
.1 & 1.522 & 1.53 \\
05 & 1809 & 1.79
\end{tabular}

Fig. 3. Stress concentration for grooved shaft.

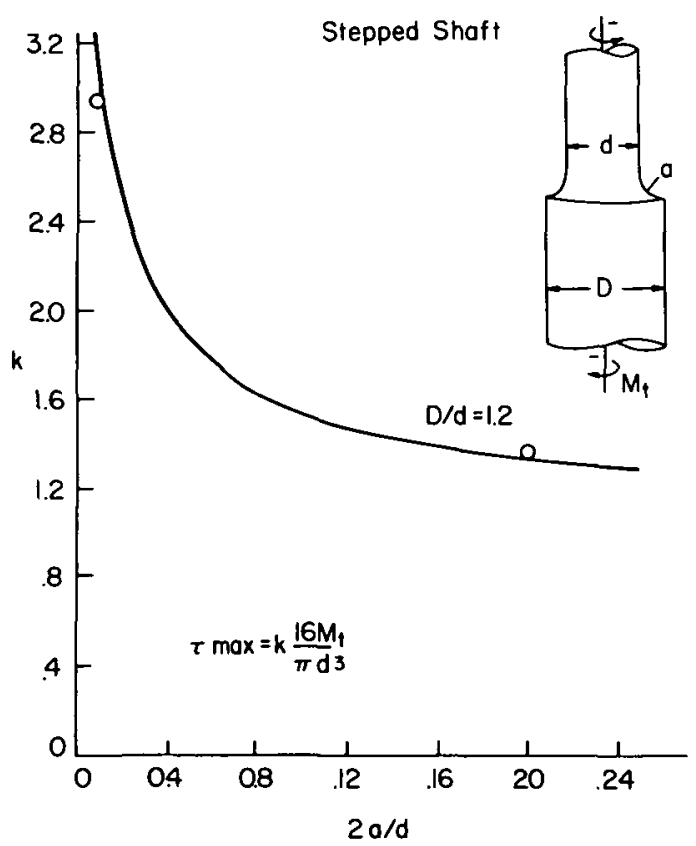

Fig. 4. Stress concentration for stepped shaft. 
The problem of a stepped shaft $D / d=1.2$ is depicted in Fig. 4 along with a curve of the variation of $k$ with $2 a / d$ from Timoshenko and Goodier [23]. Two BIE runs were made, and the results are shown as data points near the curve of Fig. 4. $N=67$ and $N=41$ nodes were used, respectively, for the higher and lower $k$ values indicated.

For scalar problems such as these, the number of simultaneous equations equals the number of nodes, and no individual computer run required more than $8 \mathrm{sec}$ CPU time.

\section{Discussion}

The BIE method is clearly limited to linear, although not necessarily selfadjoint operators $L$, and, in general, $L$ 's with variable coefficients present a comparatively formidable problem in constructing the necessary $K(x, y)$ (see [9], [24] and [3]). The fundamental solution $K_{1}(x, y) / r(y)$ of (5.1), itself in the form of an integral, is perhaps only indicative of the difficulties associated with variable coefficients in $L$.

If $L(w)=h \neq 0$ (see (2.1)) where $h$ is a prescribed function in $B$, the associated BIE of the type (2.5) contains a $B$ integral, albeit known. Such a particular integral for certain $h$ may often be conveniently converted to $S$ integrals (see [22]) by an additional application of Green's theorem.

It may be of interest to note that (2.5), with $x \in B$, is but a step away from the classical concept of representing the solution of a boundary value problem in terms of a region-dependent Green's function. For example, if a regular solution $H$ of $(2.1)$ is added to $K(x, y)$, where $H+K$ vanishes on $S$, the second of the integrals in (2.5) vanishes and the solution is given via (2.5) in terms of prescribed boundary data only. The difficulties associated with finding $H$ are sufficiently formidable usually to abandon such an approach for concrete problems.

A variation of the present formulation, related to more classical ideas (see [13] and [14]) is to recognize that each of the two integrals in (2.5) is a solution of (2.1), and it suffices to seek a solution to a given boundary value problem in the form of one such integral (for example [7]) where a density function $\phi(y)$ is the multiplier of $K(x, y)$ or $B_{p}[K(x, y)]$ in the chosen integral. In the limit as $x \in B \rightarrow x \in S$, a BIE is obtained in the unknown $\phi(y)$. This approach, frequently called the "indirect BIE method" (for example [1]), has its merit in that prescribed boundary data enter the problem directly rather than in the form of integrals. Further, integral equations of the "second kind" ([13], [6]) with better spectral characteristics arise. However, the present "direct" approach is 
more flexible for mixed boundary data, and the physical variables appear directly as unknowns. These are generally better behaved than a non-physical $\phi$ near corners and notches. Finally, in many problems for which the set $u, B_{v}(u)$, is all that is required, no integrals need be evaluated following the (numerical) solution of the BIE.

The reader familiar with the currently most popular method for the numerical solution of boundary value problems, namely the Finite Element (FE) method (for example [27]), will note certain obvious similarities. Elements and nodes are used, shape functions incorporated, etc., and the process leads to a system of linear algebraic equations involving physical quantities such as stress and displacement. Quite complex problems may be attacked with both methods, although the FE method is more readily applied to inhomogeneous (variable coefficient equations) and nonlinear problems. Indeed, the BIE method is of ten called the "Boundary Element" method ([2] and [1]) which suggests to some that the methods are basically the same except that only boundary elements are needed in the one case.

We submit that such terminology focuses too quickly on the numerical procedures of the type discussed here and clouds the essential fact that (2.5) is an exact integral restatement of the boundary value problem governed by (2.1). Such a restatement is made possible only by the existence and employment of the fundamental singular solution of the original differential equation. This solution with property (2.3) makes explicit, through (2.5), an exact boundary constraint relation among complementary boundary data $\left(u, B_{v}(u)\right)$ such that once all such data are determined, the field is given by simple quadrature. The analytical process reduces the dimension of the problem by one, and in reality "the problem is solved" on the boundary. The BIE must be solved approximately in general, and the field quadratures performed in a nonexact fashion, but elements as introduced herein are only one way to accomplish the objective. They are in no way an essential part of the process as is the case with the FE method.

The illustrative problems chosen here in $E^{3}$ and $E^{2}$ are rather far apart on the spectrum of computational difficulty as the CPU times of $193 \mathrm{sec}$ versus $8 \mathrm{sec}$ suggest. The square matrices $A$ and $B$ in (3.4) are full in every case and most of the CPU time, for problems of the present size, is taken generating the matrix elements. The contrast, then, between a vector problem in $E^{3}$ versus a scalar one in $E^{2}$ is apparent.

Square matrices in the FE method are banded, so despite their generally larger size based on a volume (area) versus surface (curve) for the BIE method, for problems in $E^{3}$ and $E^{2}$, respectively, there is a trade-off in efficiency. Nevertheless, there is usually a computational advantage with the BIE method 
(see for example [1] and [4]), especially for problems in which high gradients of the relevant function occur on or near the boundary, such as in the presented examples. Coarser meshes are possible with the BIE, leading to smaller (albeit full) matrices, lower CPU times, not to mention the advantages of dealing with a surface rather than a full-domain meshwork. However, the mushrooming capability in computer graphics and automatic mesh generation is beginning to be an enormous convenience for both methods. Nevertheless, aside from such tactical but often economically important questions of data handling, a key issue for the analyst is the realization that the BIE represents an exact integral restatement of a problem. The role played by $K(x, y)$ has no counterpart with field methods, and BIE methods should, in fact, be computationally superior for problems where it can be used.

Modern methods of numerical analysis continue to allow errors with the BIE method to be made very small indeed. Crude discretization procedures such as piecewise constant or linear surface representation have given way to the presently described quadratic isoparametric procedure. Cubic representations [25] and Hermitian Cubics as well as spline representations [26] have been and are still being developed. All are essentially collocation methods, but successive-approximations or least-squares techniques can clearly be used as well. However, a systematic error analysis for the BIE method based on any approximation/discretization scheme remains to be performed. In any case, the BIE method, by itself or through its "marriage" with the FE method, seems to be emerging as an important numerical solution method for many practical problems.

\section{Acknowledgement}

Thanks are due A. K. Gupta of ITT Grinnell Corporation for gathering some numerical data, and G. Fairweather of the University of Kentucky for a valuable conversation.

\section{Appendix}

Kelvin Solution $\left(E^{3}\right)$

$U(x, y)=\frac{(1+\sigma)}{8 \pi E(1-\sigma)}\left(\frac{1}{r(x, y)}\right)[(3-4 \sigma) 1+\operatorname{grad} r(x, y) \otimes \operatorname{grad} r(x, y)]$, 
in which $r(x, y)=|y-x|, 1$ is the identity tensor, $\otimes$ represents the tensor product,

$$
E=\frac{\mu(3 \lambda+2 \mu)}{\lambda+\mu} \quad \text { and } \quad \sigma=\frac{\lambda}{2(\lambda+\mu)}
$$

\section{Torsion Fundamental Solution}

$$
K_{1}(x, y) / r(y)=\int_{-\pi}^{\pi} \frac{\cos \theta d \theta}{R},
$$

where $R=\left[r^{2}(y)+r^{2}(x)-2 r(y) r(x) \cos \theta+(z(y)-z(x))^{2}\right]^{1 / 2}$.

$$
\hat{K}_{1}=r(y) \frac{\partial}{\partial \nu_{y}}\left(K_{1}(x, y) / r(y)\right)-\frac{\partial r(y)}{\partial \nu_{y}}\left(K_{1}(x, y) / r(y)\right) \text {. }
$$

\section{References}

[1] P. K. Banerjee and R. Butterfield, Boundary elements in engineering science (McGraw-Hill, London, 1980).

[2] C. Brebbia, The boundary element method for engineers (John Wiley, New York, 1978).

[3] D. L. Clements, "A boundary integral equation method for the numerical solution of a second order elliptic equation with variable coefficients," J. Australian Math. Society, B, Appl. Math. (to appear).

[4] T. A. Cruse and F. J. Rizzo eds. "Boundary integral equation method: computational applications in applied mechanics," $A S M E A M D$-Vol. 11 (1975), 1-141.

[5] G. Fairweather, F. J. Rizzo, D. J. Shippy and Y. Wu, "On the numerical solution of two-dimensional potential problems by an improved boundary integral equation method," $J$. Computational physics 31, 1 (1979), 96-112.

[6] U. Heise, "The spectra of some integral operators for plane elastostatical boundary value problems," J. elasticity 8 (1978), 47-49.

[7] J. L. Hess, "Review of integral equation techniques for solving potential flow problems with complicated boundaries," Proc. Second Intl. Symp. Innovative numerical analysis in applied engineering science (Virginia U. Press, Charlottesville, 1980), 131-143.

[8] M. A. Jaswon, "Integral equation methods in potential theory. I", Proceedings of the Royal Society 273, A (1963), 23-32.

[9] F. John, Plane waves and spherical means (Interscience, New York, first edition, 1955).

[10] O. D. Kellogg, Foundations of potential theory (Dover, New York, first edition, 1953).

[11] J. C. Lachat and J. O. Watson, "Effective numerical treatment of boundary integral equations: A formulation for three-dimensional elastostatics", Int. J. Numerical methods in engineering 10, 5 (1976), 991.

[12] A. E. H. Love, $A$ treatise on the mathematical theory of elasticity (Dover, New York, fourth edition, 1944).

[13] W. V. Lovitt, Linear integral equations (Dover, New York, 1950).

[14] N. I. Muskhelishvili, Singular integral equations (Noordhoff, Holland, 1953).

[15] R. E. Peterson, Stress concentration factors (Wiley, New York, 1974).

[16] F. J. Rizzo, "An integral equation approach to boundary value problems of classical elastostatics", Quarterly of applied mathematics 25, 1 (1967), 83-95. 
[17] F. J. Rizzo, A. K. Gupta and Y. Wu, "A boundary integral equation method for torsion of variable diameter circular shafts and related problems," Proc. Second Intl. Symp. Innovative numerical anabysis in applied engineering science (Virginia U. Press, Charlottesville, 1980), 373-380.

[18] F. J. Rizzo and D. J. Shippy, "An advanced boundary integral equation method for three-dimensional thermoelasticity", Int. J. Numerical methods in engineering 11, (1977), 1753-1768.

[19] K. R. Rushton, "Stress concentrations arising in the torsion of grooved shafts," Intl. J. Mechanical science 9 (1967), 697-705.

[20] R. P. Shaw, "BIE methods applied to wave problems", Developments in boundary element methods 1 (1979), 121-153.

[21] I. Stakgold, Boundary value problems of mathematical physics-volume 1 (Macmillan, New York, 1967).

[22] M. Stippes and F. J. Rizzo, "A note on the body force integral of classical elastostatics," ZAMP 28 (1977), 339-341.

[23] S. Timoshenko and J. N. Goodier, Theory of elasticity (MCGraw-Hill, New York, 1951).

[24] I. N. Vekua, New methods for solving elliptic equations (North-Holland, Netherlands, 1967).

[25] J. O. Watson, "Hermitian cubic boundary elements for plane elastostatics", Proc. Second Intl. Symp. Innovative numerical anabysis in applied engineering science (Virginia U. Press, Charlottesville, 1980), 403-412.

[26] Y. Wu, "The boundary integral equation method using various approximation techniques for problems governed by Laplace's equation", U. S. Air Force Special Report AFOSR-TR-761313, Washington, DC, (1976).

[27] O. C. Zienkiewicz, The finite element method (McGraw-Hill, London, third edition, 1977).

\section{Department of Engineering Mechanics}

University of Kentucky

Lexington

Kentucky 40506

U.S.A. 